\title{
Single quantum emitter Dicke enhancement
}

\author{
Tommaso Tufarelli $\odot,{ }^{1, *}$ Daniel Friedrich, ${ }^{2}$ Heiko Groß, ${ }^{2}$ Joachim Hamm, ${ }^{3}$ Ortwin Hess $\odot,{ }^{3,4, \dagger}$ and Bert Hecht $\odot^{2, \sharp}$ \\ ${ }^{1}$ School of Mathematical Sciences and Centre for the Mathematics and Theoretical Physics of Quantum Non-Equilibrium Systems, \\ University of Nottingham, Nottingham NG7 2RD, United Kingdom \\ ${ }^{2}$ Nano-Optics and Biophotonics Group, Experimentelle Physik 5, Physikalisches Institut, Universität Würzburg, D-97074 Würzburg, Germany \\ ${ }^{3}$ The Blackett Laboratory, Department of Physics, Imperial College London, London SW7 2AZ, United Kingdom \\ ${ }^{4}$ School of Physics and CRANN Institute, Trinity College Dublin, Dublin 2, Ireland
}

(Received 29 October 2020; revised 25 March 2021; accepted 2 June 2021; published 29 July 2021)

\begin{abstract}
Coupling $N$ identical emitters to the same field mode is a well-established method to enhance light-matter interaction. However, the resulting $\sqrt{N}$ boost of the coupling strength comes at the cost of a "linearized" (effectively semiclassical) dynamics. Here, we instead demonstrate a new approach for enhancing the coupling constant of a single quantum emitter, while retaining the nonlinear character of the light-matter interaction. We consider a single quantum emitter with $N$ nearly degenerate transitions that are collectively coupled to the same field mode. We show that in such conditions an effective Jaynes-Cummings model emerges with a boosted coupling constant of order $\sqrt{N}$. The validity and consequences of our general conclusions are analytically demonstrated for the instructive case $N=2$. We further observe that our system can closely match the spectral line shapes and photon autocorrelation functions typical of Jaynes-Cummings physics, proving that quantum optical nonlinearities are retained. Our findings match up very well with recent broadband plasmonic nanoresonator strong-coupling experiments and will, therefore, facilitate the control and detection of single-photon nonlinearities at ambient conditions.
\end{abstract}

DOI: 10.1103/PhysRevResearch.3.033103

\section{INTRODUCTION}

Strongly coupled light-matter systems, described by the Jaynes-Cummings (JC) model [1], are of fundamental interest in testing the quantized nature of coupled light and matter degrees of freedom in the context of cavity QED [2]. Importantly, JC physics requires that a single two-level emitter interacts with a single-mode electromagnetic field. Such systems can be relevant for quantum information processing, for example, in interfacing flying and static qubits [3] or for quantum-state engineering [2-6]. Yet strong coupling of light and a single emitter is not easy to achieve in practice, due to the large mismatch in the spatial extension of free-space photons and typical emitters (i.e., artificial or real atoms) that feature electronic transitions in the optical domain $[7,8]$.

A possible route to overcome this difficulty is to engineer an "effective" emitter with a large dipole moment. This is most commonly implemented by combining $N \gg 1$ identical emitters coupled to the same field, resulting in the wellknown "Dicke enhancement" of light-matter interaction: The effective coupling strength is boosted by a factor of order $\sqrt{N}$, compared to the single-emitter case [9-12]. However,

\footnotetext{
*tommaso.tufarelli@nottingham.ac.uk

†ortwin.hess@tcd.ie

"hecht@physik.uni-wuerzburg.de
}

Published by the American Physical Society under the terms of the Creative Commons Attribution 4.0 International license. Further distribution of this work must maintain attribution to the author(s) and the published article's title, journal citation, and DOI. the relevant theory in such systems is the many-emitter limit of the Tavis-Cummings (TC) model [13], whose low-energy dynamics is effectively linear, i.e., indistinguishable from that of coupled harmonic oscillators [14].

The key achievement of our paper is to bypass this seemingly inevitable trade-off between coupling strength and (quantum) nonlinearity: We open an innovative route to a "Dicke-like" enhancement of the coupling constant which preserves the precious quantum optical nonlinearities of the JC model.

In this paper we indeed show how the exploitation of a multilevel emitter, with $N$ nearly degenerate excited states coupled to the same field mode, increases the effective lightmatter coupling constant by a factor of order $\sqrt{N}$. Although this is the same scaling found in the Dicke [15] and TC models [13] with $N$ emitters, the crucial difference is that in our case the quantum nonlinearity of the interaction is preserved: We demonstrate this based on emission spectra and second-order photon autocorrelation functions. More precisely, our system can closely approximate the behavior of a JC model, in principle, for arbitrary values of $N$. These findings, in their simplest form, are schematized in Fig. 1.

Additionally, we find that our predictions are robust against typical sources of decoherence (such as cavity decay and emitter dephasing) that are common at elevated temperatures. Even though our results are fully general and independent of the physical implementation of the model, we anticipate that our approach can be particularly useful in the context of room-temperature strong coupling. We, thus, find it useful to provide some context on the current state of the art of this research field and to highlight how our research can help it move forward. 

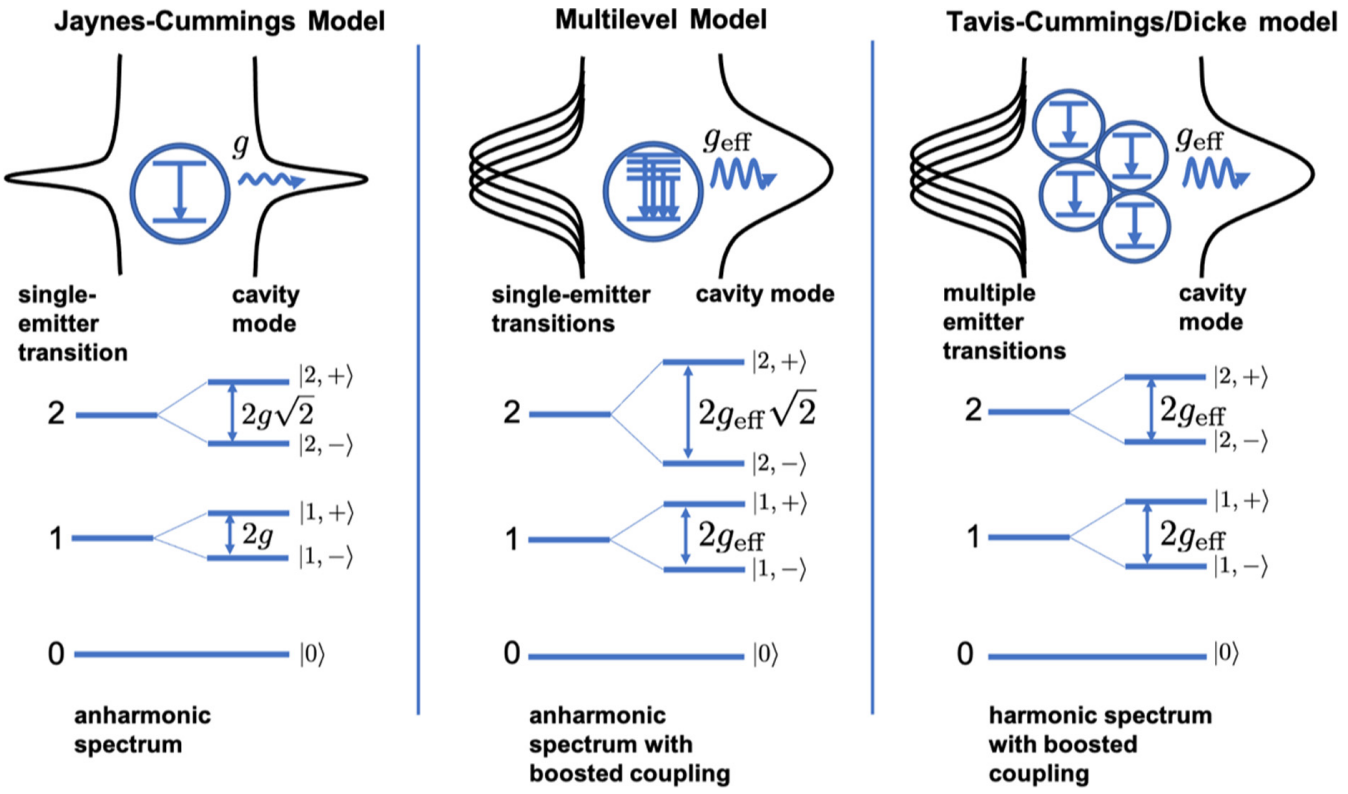

FIG. 1. Visual summary of the light-matter interaction models treated here. For multilevel, TC, and Dicke models, only energy levels that have a direct correspondence in the Jaynes-Cummings model (JCM) are shown. For the TC model, the limit $N \gg 1$ is considered. Left: Standard Jaynes-Cummings model in which a single two-level emitter couples resonantly to a single-mode field with strength $g$. The resulting anharmonic spectrum is a cornerstone of modern quantum optics but challenging to probe experimentally: The required regime $g \gg \kappa$ is difficult to achieve, where $\kappa$ is the cavity decay rate. Center: Multilevel model, the focus of this paper. A quasidegenerate multilevel emitter with $N$ closely spaced excited states interacts with a single-mode field. When all the transitions are approximately resonant with the field, an effective coupling constant $g_{\text {eff }}$, enhanced by a factor of order $\sqrt{N}$, is established. In principle, the resulting low-energy spectrum can closely approximate the JC spectrum for any value of $N$. Right: Tavis-Cummings and Dicke models in which $N \gg 1$ nearly identical two-level systems interact with the same field. A boosted effective coupling constant $g_{\text {eff }} \propto \sqrt{N}$ is established also in this case, but the spectrum becomes approximately harmonic as $N$ is increased.

Recently, room-temperature strong coupling with many emitters [16-19] and even single quantum emitters [20-24] have been experimentally demonstrated. These experiments rely on the remarkable coupling strengths achievable in plasmonic nanoresonators, thanks to the deeply subwavelength mode volumes of the latter [43-47]. In order to relax the mode volume requirements (which are currently pushing the limits of nanofabrication techniques) and obtain more robust experimental realizations, it would be of great interest to find emitter systems with increased dipole moments. This would result in larger coupling constants while still allowing to harness the single-emitter quantum nonlinearity of the JCM $[25,26]$. So far, only the "first rung" of the JC ladder, i.e., the single-excitation regime of the model, has been experimentally detected at ambient conditions. Efforts are still ongoing to detect higher rungs of the ladder to shed light on (and harness) the quantum nonlinearity of the model.

In order to realize the JCM experimentally, great efforts have been made over the years in the fabrication of artificial atoms — such as semiconductor quantum dots [27] — that are close to ideal two-level emitters. Indeed, so far the multilevel nature of such emitters has mainly been treated as a modeling nuisance, i.e., as a source of errors with respect to an idealized two-level scenario. In this context, Madsen et al. [28] and our previous paper [22] have recently mentioned the possible effects of a more complicated energy-level structure of the emitter. Additionally, our previous work [22] explicitly suggested that an emitter with multiple $(>2)$ energy levels can even bring about some advantages: In the right conditions it may be used to simulate a JC system with an enhanced coupling constant. With reports of quantum dots featuring as much as 64 quasidegenerate excited states [29,30], a general quantum-mechanical analysis of such a system (multilevel model for brevity) is in order.

Therefore, the findings presented here will be of importance for the selection and the design of suitable emitters for nanoscale strong-coupling experiments at ambient conditions and for the interpretation of such experiments. Importantly, our approach lives independently of the contentious issues regarding the calculation of mode volumes and coupling constants in quantum (and classical) nanophotonics, see, for example, Refs. [31-33]. These approaches will, of course, determine the numerical values of coupling constants in different experimental situations, but they do not affect the nature of our quantum optical model and the general conclusions we can draw from it.

The paper is organized as follows. In Sec. II we introduce a Hamiltonian that models an emitter with $N$ excited levels coupled to the same ground state via a single-mode field. We will show that our system can be understood as a JC model which is weakly interacting with $N-1$ "dark states" of the emitter. In Sec. III we generalize our model to an open system via a master equation. This takes into account incoherent processes, such as photon loss, dephasing, and emitter pumping, all of which may be required for describing realistic experimental scenarios. In Sec. IV we support our general arguments with 
detailed analytical results for the special case $N=2$ : We find that our system's emission spectrum features approximately the same resonances as a JC model, plus additional features (due to the dark states) that we fully characterize. Section V presents numerical studies for $N=3$, comparing common experimental observables in our model with the same quantities calculated in reference JC and TC/Dicke models. In Sec. VI we draw our conclusions. For completeness, the Appendix contains some useful reminders and formulas pertaining to JC, TC, and coupled-oscillator models, since those are referenced throughout the paper.

\section{MODEL}

Our multilevel model features a quantum emitter with electronic ground-state $|G\rangle$ and a collection of excited states $\left|e_{k}\right\rangle$, also called sublevels, where $k=1,2, \ldots, N$. Each internal transition of the emitter $|G\rangle \leftrightarrow\left|e_{k}\right\rangle$ is coupled to a single-mode field (cavity for brevity) with strength $g_{k} \in \mathbb{R}$. The typical physical system we have in mind is a multilevel quantum dot embedded in a plasmonic nanoresonator where the cavity resonance is broadband, in the sense that it overlaps with all the emitter transitions. Additionally, the strong field gradients of nanoresonators may allow for light-matter couplings beyond the dipole approximation, for example by activating quadrupole-allowed transitions of the emitter. Hence, this is a scenario where the field can potentially interact with a larger number of emitter levels as compared to the standard cavity-QED setup which is typically restricted to dipole transitions. Yet, it is important to note that our quantum optical formalism applies to any type of emitter or resonator that satisfies our modeling assumptions.

As usual, the cavity mode is described via the annihilation operator $\hat{a}$ with $\left[\hat{a}, \hat{a}^{\dagger}\right]=1$. Taking $\hbar=1$ for convenience, the cavity resonance has average frequency (hence, energy) $\omega_{0}$, the energy of $|G\rangle$ can be set to zero without loss of generality, whereas each state $\left|e_{k}\right\rangle$ has energy $\omega_{0}+\Delta_{k}$. Here $\Delta_{k}$ is the detuning between the $k$ th emitter transition $\left(\left|e_{k}\right\rangle \leftrightarrow\right.$ $|G\rangle)$ and the cavity. For convenience we assume $\Delta_{1} \leqslant \Delta_{2} \leqslant$ $\cdots \leqslant \Delta_{N}$; we indicate the full range of energy detunings as $\varepsilon \equiv \Delta_{N}-\Delta_{1}$ and the average detuning as $\Delta \equiv \frac{1}{N} \sum_{k} \Delta_{k}$. We can write the total Hamiltonian as

$$
\begin{aligned}
H & =H_{0}+V, \\
H_{0} & =\omega_{0} \hat{a}^{\dagger} \hat{a}+\sum_{k=1}^{N}\left(\omega_{0}+\Delta_{k}\right)\left|e_{k}\right\rangle\left\langle e_{k}\right|, \\
V & =\sum_{k=1}^{N} g_{k}\left(\hat{a}\left|e_{k}\right\rangle\left\langle G\left|+\hat{a}^{\dagger}\right| G\right\rangle\left\langle e_{k}\right|\right),
\end{aligned}
$$

where $H_{0}, V$ are, respectively, the free and interaction Hamiltonians and we have neglected counter-rotating and diamagnetic terms in the light-matter interaction. Such approximations are justified provided that the overall coupling constant $g_{\text {eff }}$ (defined below) is significantly smaller than the "bare" frequency $\omega_{0}$ [34]. In passing we do note that recent experiments in nanoplasmonic QED are pushing the limits of validity of this assumption as, for example, $g_{\text {eff }} \simeq 0.07 \omega_{0}$ in Ref. [22]. Therefore, in future work it will be interesting to (a) Bare emitter basis

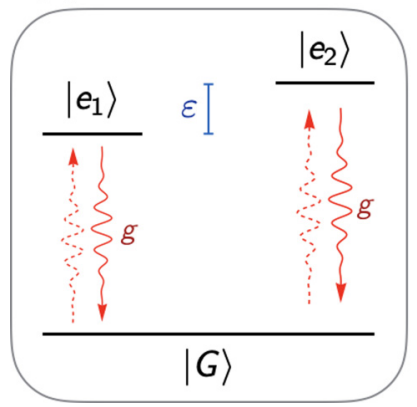

(b) Radiation basis

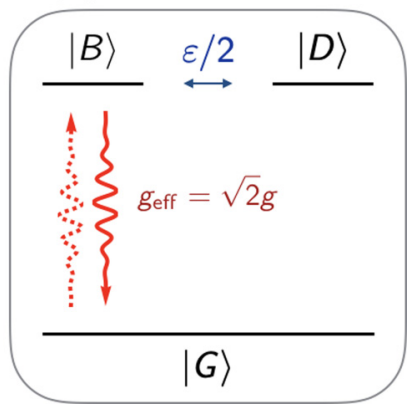

FIG. 2. Illustration of the transformation between the bare emitter basis and the radiation basis, for $N=2$ sublevels, and the resulting appearance of a boosted effective coupling. The same principles hold for larger values of $N$. (a) In the bare emitter basis the two levels $\left|e_{1,2}\right\rangle$ are not directly coupled to each other, whereas $\varepsilon$ plays the role of a relative detuning between them. Both $|G\rangle \leftrightarrow\left|e_{1,2}\right\rangle$ transitions couple to the cavity field, and we are assuming for simplicity that the associated coupling constants are the same: $g_{1}=g_{2}=g$. (b) In the radiation basis, the bright- and dark-states $|B\rangle,|D\rangle$ have the same frequency but are coupled to each other with strength $\varepsilon / 2$. The transition $|G\rangle \leftrightarrow|B\rangle$ is coupled to the field with enhanced strength $g_{\text {eff }}=\sqrt{2} g$, whereas the dark-state $|D\rangle$ does not directly interact with the field.

extend our theory to the ultra-strong-coupling regime where $g_{\text {eff }} \gtrsim 0.1 \omega_{0}[34]$.

The physics of Hamiltonian (1) can, in general, be quite complicated, despite $H$ being number conservingi.e., $H$ commutes with the number operator $\hat{\mathcal{N}}_{\text {tot }}=\hat{a}^{\dagger} \hat{a}+$ $\sum_{k}\left|e_{k}\right\rangle\left\langle e_{k}\right|$. However, here we are interested in the special case of quasidegenerate emitters that are near resonant with the cavity, i.e., we assume the parameter regime $\left|\Delta_{k}\right| \ll g_{k}$. This approximation holds, e.g., for colloidal quantum dots coupled to plasmonic nanoresonators. In such a case, $\Delta_{k}$ are of the order of a few meV, whereas the coupling strengths $g_{k}$ are of the order of $100 \mathrm{meV}$ [22]. In this scenario a number of qualitative observations can be made.

\section{A. Bare emitter basis versus radiation basis}

To aid understanding, the main ideas behind this subsection are illustrated in Fig. 2 for the simplest case $N=2$. We begin our study of the general $N$-sublevel model by considering the case of exact degeneracy of the excited states, $\Delta_{k}=\Delta \forall k$, or, equivalently, $\varepsilon=0$ in terms of the full range of energy detunings. The resulting mathematics follow in a straightforward manner from the well-known JCM. This simple starting point will set the stage to investigate small deviations from an idealized scenario, i.e., cases with small but nonzero $\varepsilon$. For $\varepsilon=0$, we find that any linear combination of the states $\left|e_{k}\right\rangle$ is itself a valid emitter excited state, or more precisely, an eigenstate of $H_{0}$ with eigenvalue $\omega_{0}+\Delta$. Among all the states that can be constructed in this way, of particular significance is the coherent superposition,

$$
|B\rangle=\frac{\sum_{k} g_{k}\left|e_{k}\right\rangle}{\sqrt{\sum_{j} g_{j}^{2}}},
$$


where the denominator ensures normalization. We will refer to $|B\rangle$ as the bright state or superradiant state (mimicking the terminology of the Dicke model). State $|B\rangle$ can be interpreted as the only emitter excited state that couples directly to the cavity field. Indeed it is easy to check that the light-matter interaction term in Eq. (1) may be rewritten in the simple form

$$
V=g_{\text {eff }}\left(\hat{a}|B\rangle\left\langle G\left|+\hat{a}^{\dagger}\right| G\right\rangle\langle B|\right),
$$

where we have defined an effective coupling constant,

$$
g_{\text {eff }}=\sqrt{\sum_{k} g_{k}^{2}} \text {. }
$$

Hence, through an appropriate change of basis for the emitter, the $N$-degenerate transitions $|G\rangle \leftrightarrow\left|e_{k}\right\rangle$ can be replaced by a single transition $|G\rangle \leftrightarrow|B\rangle$, characterized by an enhanced coupling to the cavity field. Equation (6) indeed shows that the effective coupling features the scaling $g_{\text {eff }} \sim \sqrt{N}$ with the number of sublevels. This boosting of the effective lightmatter coupling is analogous to what happens in the Dicke [15] and TC [13] models, where $N$ identical emitters are coupled to the same field. A crucial difference from the TC model is that Eq. (5) retains the structure of a JC interaction Hamiltonian so that in the model studied here there is, in principle, no loss of anharmonicity as the number of sublevels $N$ is increased.

To fully specify the mentioned change of basis, we can define $N-1$ further linear combinations of the excited states $\left|e_{k}\right\rangle$, say $\left\{\left|D_{1}\right\rangle,\left|D_{2}\right\rangle, \ldots,\left|D_{N-1}\right\rangle\right\}$, which together with $\{|G\rangle,|B\rangle\}$ form a complete orthonormal set for the emitter internal levels. When $\varepsilon=0$ each $\left|D_{k}\right\rangle$ is also an eigenstate of $H_{0}$ with eigenvalue $\omega_{0}+\Delta$, due to the $N$-fold degeneracy. By construction, these additional basis states do not directly interact with the cavity field, i.e.,

$$
V\left|D_{k}\right\rangle=\left\langle D_{k}\right| V=0, \quad k=1,2, \ldots, N-1,
$$

and may be called subradiant states or dark states. In practice, explicit expressions for the dark states may be found by standard orthogonalization procedures (details not shown). The above discussion suggests that the set $\left\{|G\rangle,|B\rangle,\left|D_{1}\right\rangle,\left|D_{2}\right\rangle, \ldots,\left|D_{N-1}\right\rangle\right\}$, which we will call the $r a-$ diation basis, may be a more meaningful conceptual tool to study light-matter interaction in our system as compared to the bare emitter basis $\left\{|G\rangle,\left|e_{1}\right\rangle,\left|e_{2}\right\rangle, \ldots,\left|e_{N}\right\rangle\right\}$.

For the purpose of modeling the optical properties of the system, it is now tempting to simply neglect all the dark states and retain only the two emitter levels $|B\rangle,|G\rangle$. This is justified if there are no additional processes, coherent or incoherent, that are able to populate the dark states and/or couple them to the two levels $|B\rangle,|G\rangle$. When all these conditions are satisfied, the system is exactly described by a JCM with coupling constant $g_{\text {eff }}$.

We are now in a position to study small deviations from the ideal case. As anticipated, the most obvious deviation from pure JC physics occurs when the excited-states $\left|e_{k}\right\rangle$ are not exactly resonant with each other (i.e., $\varepsilon \neq 0$ ). In the radiation basis, the (small) relative detunings between the levels $\left|e_{k}\right\rangle$ will translate into (weak) couplings between the bright and the dark states and between the dark states themselves. In other words, $|B\rangle$ and $\left|D_{k}\right\rangle$ cease to be eigenstates of $H_{0}$ when $\varepsilon \neq 0$.
Note, however, that Eqs. (5) and (7) remain valid in general, so that the dark states may interact with the field only indirectly, i.e., through the mediation of state $|B\rangle$. These additional interactions will bring about deviations from the well-understood JC physics described above. Although a general calculation of these effects would be cumbersome and beyond the scope of this paper, the case $N=2$ sublevels is simple enough to be treated analytically and already contains all the essential ingredients in our problem-see Sec. IV below and Fig. 2.

In some cases, the exact equivalence to a JC system may be lost even in the case of perfect degeneracy between the excited sublevels. This can happen if incoherent processes couple the pair $\{|G\rangle,|B\rangle\}$ to the dark states. We will see in Sec. IV, for example, that emitter dephasing provides a common mechanism for interconverting bright and dark states. Yet, our analytical and numerical results suggest that the multilevel model can provide an excellent approximation to JC physics even when these imperfections are taken into account.

\section{MASTER EQUATION}

In order to predict relevant observables for future experiments, we now introduce an open system generalization of our model, via the well-established Gorini-KossakowskiSudarshan-Lindblad (GKSL) master equation [35]. As anticipated above, the main experimental scenario we have in mind is a quantum dot interacting with a plasmonic nanoresonator, yet the ideas presented here may be applicable to a wider variety of setups. We will include cavity photon loss as the primary channel for energy dissipation, whereas the decay rate of the emitter into free-space modes $\gamma$ will be assumed negligible. Such an assumption is reasonable due to the large $\beta$ factors achievable in plasmonic resonators. For a brief discussion on the effects of small but nonzero $\gamma$, see Ref. [36]. On the other hand, we will assume that the emitter excited states can be broadened well beyond their radiative linewidth by additional dephasing processes-typically thought of as a drawback of room-temperature operation.

We consider two ways in which energy can be supplied to the coupled system: incoherent pumping of the emitter or a coherent driving field applied to the cavity. Although the first is a common method to feed excitations into quantum dots, the second is arguably the standard avenue to test nonlinearities in a quantum optical system. For a visual summary, the main ingredients of our open system model are sketched in Fig. 3. In formulas, the following master equation will be used to model the emitter-cavity system,

$$
\dot{\rho}=-i\left[H+V_{\text {drive }}, \rho\right]+\sum_{L \in \mathcal{J}} \frac{1}{2}\left(2 L \rho L^{\dagger}-L^{\dagger} L \rho-\rho L^{\dagger} L\right),
$$

where $H$ is the Hamiltonian (1) and

$$
V_{\text {drive }}=\Omega\left(\hat{a} e^{i \omega_{L} t}+\hat{a}^{\dagger} e^{-i \omega_{L} t}\right)
$$

is an interaction term describing coherent driving of the cavity by a classical laser at frequency $\omega_{L}$ ( $\Omega$ quantifies the driving field amplitude). The $L$ 's appearing on the right-hand side of Eq. (8) are the jump operators, used to model incoherent processes. They are collected in set $\mathcal{J}$, which features the 


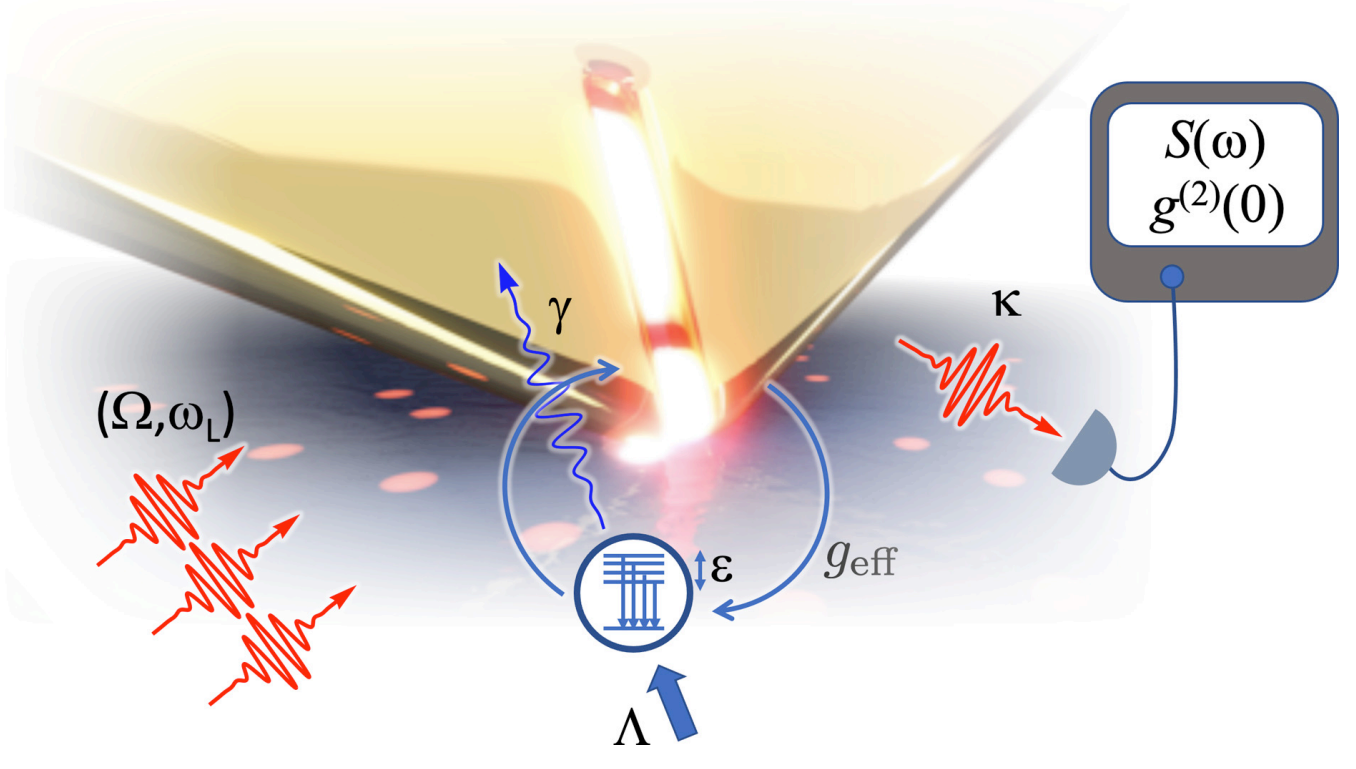

FIG. 3. Schematic of the investigated setup. The system may be excited through an incoherent pump of intensity $\Lambda$ applied to the emitter or via coherent driving of the (plasmonic) cavity with field amplitude $\Omega$ and frequency $\omega_{L}$. The bright state of the emitter is coupled to the cavity with the effective strength $g_{\text {eff }}$, and the emitter sublevels are spread in frequency over a range $\varepsilon . \kappa$ is the loss rate of the cavity, and $\gamma$ is the decay rate of all the emitter sublevels, which are also subject to dephasing at rate $\gamma_{d}$ (not shown). All the observables discussed in this paper can be measured by collecting the cavity output field.

\section{following elements:}

(i) Cavity decay (photon loss) at rate $\kappa: L=\sqrt{\kappa} \hat{a}$.

(ii) Radiative decay of the emitter $\gamma: L=\sqrt{\gamma}|G\rangle\left\langle e_{k}\right|$.

(iii) Emitter dephasing $\gamma_{d}: L=\sqrt{\gamma_{d}}\left|e_{k}\right\rangle\left\langle e_{k}\right|$.

(iv) Emitter pumping of total rate $\Lambda: L=\sqrt{\frac{\Lambda}{N}}\left|e_{k}\right\rangle\langle G|$, where $k=1,2, \ldots, N$. We assumed that the decay and dephasing rates are the same for all sublevels and that the pump power $\Lambda$ is split symmetrically over the $N$ sublevels. In reality, how much each level will be individually pumped will depend on microscopic details of the model, a study that goes beyond the scope of our present paper. Importantly, however, these details do not affect our general conclusions, provided that the total pump power is the smallest parameter of the problem: Under these conditions the locations of the spectral resonances of our coupled system are (approximately) independent of the details of the pump process (however, the relative intensities of different spectral peaks can be detail dependent in general).

We also consider equal couplings $g_{k}=g_{\text {eff }} / \sqrt{N}$ for all $k$, whereas the detunings $\Delta_{k}$ are equally spaced in the interval $[\Delta-\varepsilon / 2, \Delta+\varepsilon / 2]$. All the assumed symmetries between the sublevels are, of course, an idealization. Many generalizations of this basic model can be included in principle. However, we do not expect that these can bring about significant qualitative departures from the physics described here. Hence, in this paper we confine our analysis to the above setting for simplicity and computational efficiency.

\section{ANALYTICAL RESULTS FOR TWO SUBLEVELS}

We here present the analytical diagonalization of Hamiltonian (1) for the case $N=2$ with equal couplings $g_{1}=g_{2}=g$ as well as the associated implications for the system's open dynamics. This instance of the model, illustrated in Fig. 2, is particularly transparent and fully captures the essence of our paper. We note that for the case $N=2$ we obtain an emitter structure known as the "vee atom". Cavity QED with vee atoms was extensively investigated in the 1980s in the context of producing squeezed light and laser light with reduced phase fluctuations [37-39]. We verified that analytical solutions are also available for the cases $N=3,4$, using similar methods, but these are significantly more complex without adding fundamentally new insights. For $N=2$ the detunings are given by $\Delta_{1}=\Delta-\varepsilon / 2$ and $\Delta_{2}=\Delta+\varepsilon / 2$, respectively. Furthermore, there are only one bright and one dark state given by $|B\rangle=\frac{\left|e_{1}\right\rangle+\left|e_{2}\right\rangle}{\sqrt{2}},|D\rangle=\frac{\left|e_{1}\right\rangle-\left|e_{2}\right\rangle}{\sqrt{2}}$. In the radiation basis representation, the Hamiltonian may be recast as

$$
\begin{aligned}
H & =H_{0}^{\prime}+V^{\prime}, \\
H_{0}^{\prime} & =\omega_{0} \hat{a}^{\dagger} \hat{a}+\left(\omega_{0}+\Delta\right)(|B\rangle\langle B|+| D\rangle\langle D|), \\
V^{\prime} & =g_{\text {eff }}\left(\hat{a}|B\rangle\left\langle G\left|+\hat{a}^{\dagger}\right| G\right\rangle\langle B|\right)-\frac{\varepsilon}{2}(|B\rangle\langle D|+| D\rangle\langle B|),
\end{aligned}
$$

where in this case $g_{\text {eff }}=\sqrt{2} g$. As for the general case treated in the previous section, a JC interaction describes the coupling between the two levels $\{|G\rangle,|B\rangle\}$ and the cavity field. However, we can also see that the relative detuning of the sublevels $\varepsilon$ translates into a small coupling between $|B\rangle$ and $|D\rangle$ so that even the dark-state $|D\rangle$ can indirectly influence the optical behavior of the system. The ground state of $H$ is easily spotted as $|G, 0\rangle$ with eigenvalue $E=0$. Exploiting excitation number conservation, i.e., $\left[H, \hat{\mathcal{N}}_{\text {tot }}\right]=0$, where $\hat{\mathcal{N}}_{\text {tot }}=\hat{a}^{\dagger} \hat{a}+$ $|B\rangle\langle B|+| D\rangle\langle D|$, one can show that the remaining eigenvalues and eigenvectors of $H$ can be found by diagonalizing the $3 \times 3$ matrices $(n=1,2, \ldots, \infty)$,

$$
\uplus_{n}=\left(\begin{array}{ccc}
n \omega_{0} & g_{\text {eff }} \sqrt{n} & 0 \\
g_{\text {eff }} \sqrt{n} & n \omega_{0}+\Delta & -\varepsilon / 2 \\
0 & -\varepsilon / 2 & n \omega_{0}+\Delta
\end{array}\right),
$$



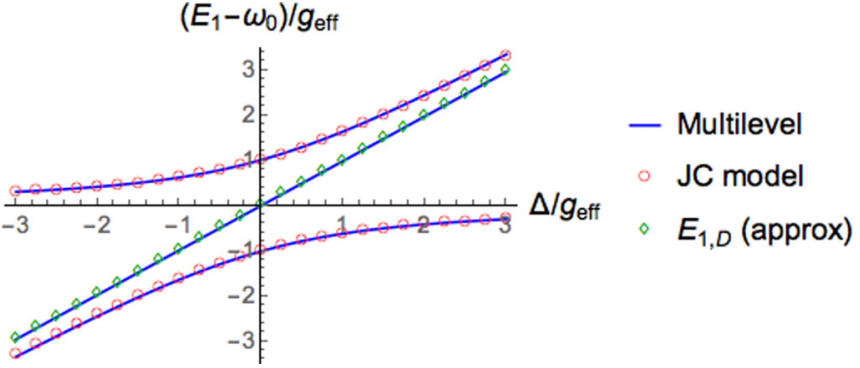

FIG. 4. Eigenvalues of $\uplus_{1}$ as a function of the average detuning $\Delta$ for $\varepsilon=0.25 g_{\text {eff }}$ (blue continuous lines). The anticrossing behavior of eigenvalues $E_{1, \pm}$ around $\Delta=0$ approximates well (the maximum relative error in the plotted range being $\sim 1.3 \%$ ) the reference JCM with coupling $g_{\text {eff }}$ (red circles). The energy $E_{1, D}$ of the quasidark state is instead approximately linear in the detuning and sits in between the JCM-like energies. An elementary perturbation theory calculation indeed yields $E_{n, D} \simeq \Delta\left(1-\varepsilon^{2} / 4 g_{\text {eff }}^{2} n\right)$, which agrees well with the exact value in the considered regime (relative error below $0.2 \%$ ). Qualitatively similar results are obtained for higher excitation numbers $n \geqslant 2$, and, in fact, it is easy to see that the relative weight of $\varepsilon$ and $\Delta$ decreases with $n$.

which describe the Hamiltonian restricted to the subspace,

$$
\left\{\hat{\mathcal{N}}_{\text {tot }}=n\right\} \equiv \operatorname{Span}\{|G, n\rangle,|B, n-1\rangle,|D, n-1\rangle\} .
$$

The behavior of the eigenvalues of $\mathrm{H}_{1}$ is shown in Fig. 4 as a function of the detuning $\Delta$. Even at the non-negligible ratio $\varepsilon / g_{\text {eff }}=0.25$ used in the figure, the commonalities with a JCM spectrum are evident. Note also that the eigenvalues of $\mathrm{H}_{n}$ for $n \geqslant 2$ will follow the same qualitative structure as a generic $\mathbb{H}_{n}$ may be obtained from $\mathrm{H}_{1}$ by a simple rescaling $g_{\text {eff }} \rightarrow g_{\text {eff }} \sqrt{n}, \omega_{0} \rightarrow n \omega_{0}$. Although a general analytical diagonalization of Eq. (13) is cumbersome, for the resonant case $\Delta=0$ one can obtain simple analytical expressions. For this case, the eigenvalues of $\uplus_{n}$ read

$$
\begin{aligned}
& E_{n, \pm}=n \omega_{0} \pm g_{\mathrm{eff}} \sqrt{n+\frac{\varepsilon^{2}}{4 g_{\mathrm{eff}}^{2}}}, \\
& E_{n, D}=n \omega_{0} .
\end{aligned}
$$

The corresponding normalized eigenvectors $\left|E_{n, \pm}\right\rangle,\left|E_{n, D}\right\rangle$ are

$$
\begin{aligned}
\left|E_{n, \pm}\right\rangle= & \frac{1}{\sqrt{2}}\left(\frac{2 g_{\mathrm{eff}} \sqrt{n}}{\sqrt{4 g_{\mathrm{eff}}^{2} n+\varepsilon^{2}}}|G, n\rangle \pm|B, n-1\rangle\right) \\
& -\frac{\varepsilon}{\sqrt{8 g_{\mathrm{eff}}^{2} n+2 \varepsilon^{2}}}|D, n-1\rangle, \\
\left|E_{n, D}\right\rangle= & \frac{\varepsilon}{\sqrt{4 g_{\mathrm{eff}}^{2} n+\varepsilon^{2}}}|G, n\rangle+\frac{2 g_{\mathrm{eff}} \sqrt{n}}{\sqrt{4 g_{\mathrm{eff}}^{2} n+\varepsilon^{2}}}|D, n-1\rangle .
\end{aligned}
$$

The above expressions suggest that in the regime $\varepsilon \ll g_{\text {eff }}$ it is natural to identify $\left\{\left|E_{n, \pm}\right\rangle, E_{n, \pm}\right\}$ as a weakly perturbed $\mathrm{JC}$ eigensystem (the latter is indeed recovered exactly for $\varepsilon \rightarrow 0)$. For brevity, we refer to those as the "JCM-type" eigenvalues and eigenvectors. The eigenstates $\left|E_{n, D}\right\rangle$, which instead are associated with the same energy levels of an empty cavity, may be understood as dressed quasidark states of the emitter, accompanied by approximately $n-1$ cavity photons. They indeed result from a hybridization of states $|G, n\rangle$ and $|D, n-1\rangle$, the second state being dominant in the superposition. It is interesting to note that the two states involved in such a superposition are only coupled indirectly in the Hamiltonian, through the mediation of state $|B, n-1\rangle$.

Exploiting the analytical results obtained here, it is possible to calculate the Hamiltonian dynamics of a generic initial state via standard methods. In fact, although our paper focuses on the regime $\varepsilon \ll g_{\text {eff }}$, the above diagonalization procedure is valid for a general choice of the model parameters.

\section{A. Qualitative analysis of the master equation for $N=2$}

The closed expressions obtained so far are particularly helpful in analyzing the open system behavior of our multilevel model, as well as its relationship to the JC model. Since in this paper we focus on the regime of low pumping (or low coherent driving) and negligible emitter decay, we can gain valuable intuition about the properties of the master equation (8) by setting $\gamma=\Lambda=\Omega=0$. First, we observe that the introduction of a photon loss rate $\kappa$ results in a finite lifetime for the excited states of $H$, such that eigenstates with $\hat{\mathcal{N}}_{\text {tot }}=n$ will tend to decay towards states with $\hat{\mathcal{N}}_{\text {tot }}=n-1$, until the ground-state $|G, 0\rangle$ is finally reached. For a given eigenstate $\left|E_{j}\right\rangle$, an estimate of the decay rate induced by photon loss can be obtained via the perturbation theory formula,

$$
\Gamma_{j} \simeq \kappa\left\langle E_{j}\left|\hat{a}^{\dagger} \hat{a}\right| E_{j}\right\rangle,
$$

which at second order in $\varepsilon$ yields $(n \geqslant 1)$,

$$
\begin{aligned}
& \Gamma_{n D} \simeq \kappa\left(n-1+\frac{\varepsilon^{2}}{4 n g_{\text {eff }}^{2}}\right), \\
& \Gamma_{n \pm} \simeq \kappa\left(n-\frac{1}{2}-\frac{\varepsilon^{2}}{8 n g_{\text {eff }}^{2}}\right) .
\end{aligned}
$$

We may immediately note that the lowest quasi-dark-state $\left|E_{1 D}\right\rangle$ is long lived, whereas all the remaining excited states have approximately the same lifetime of either the JCM excited states or the Fock states of an empty and lossy cavity. Next, emitter dephasing will induce further broadening of the Hamiltonian energy levels, which can be estimated as

$$
\delta E_{j} \simeq \gamma_{d}\left(\left|\left\langle e_{1} \mid E_{j}\right\rangle\right|^{2}+\left|\left\langle e_{2} \mid E_{j}\right\rangle\right|^{2}\right) .
$$

Some straightforward algebra yields

$$
\begin{aligned}
& \delta E_{n D} \simeq \gamma_{d}\left(1-\frac{\varepsilon^{2}}{4 n g_{\text {eff }}^{2}}\right), \\
& \delta E_{n \pm} \simeq \frac{\gamma_{d}}{2}\left(1+\frac{\varepsilon^{2}}{4 n g_{\text {eff }}^{2}}\right),
\end{aligned}
$$

where again we have expanded our results at second order in $\varepsilon$. In this case we can see that the quasidark states are the most affected, since they feature a larger overlap with the bare excited states of the emitter. Note also that the $\delta E_{j}$ 's are not associated with a decay towards states of lower excitation number (more below). The exact broadening of the energy levels, induced by the combined effect of photon loss and 
emitter dephasing, can be obtained as $-2 \operatorname{Im}\left(\tilde{E}_{j}\right)$, where the $\tilde{E}_{j}$ 's are the eigenvalues of the non-Hermitian operator,

$$
\tilde{H}=H-i \frac{\kappa}{2} \hat{a}^{\dagger} \hat{a}-i \frac{\gamma_{d}}{2} \sum_{k}\left|e_{k}\right\rangle\left\langle e_{k}\right| .
$$

The resulting expressions are unwieldy, however, we checked via a numerical optimization that $-2 \operatorname{Im}\left(\tilde{E}_{j}\right) \simeq \Gamma_{j}+\delta E_{j}$ with a relative error below $\simeq 3.1 \%$ in the ranges $0 \leqslant \varepsilon \leqslant$ $0.25 g_{\text {eff }}, 0 \leqslant \kappa \leqslant 2 g_{\text {eff }}, 0 \leqslant \gamma_{d} \leqslant 2 g_{\text {eff }}$, which is more than enough for our scope.

In order to gain qualitative understanding of photon emission processes in our system, we next look at the (squared) matrix elements of the annihilation operator $\hat{a}$,

$$
A_{i \rightarrow f}=\left|\left\langle E_{f}|\hat{a}| E_{i}\right\rangle\right|^{2},
$$

between an initial energy eigenstate $\left|E_{i}\right\rangle$ and a final one $\left|E_{f}\right\rangle$. The quantum jump approach to GKSL master equations [35] provides a useful interpretation for these quantities: $A_{i \rightarrow f}$ is proportional to the probability that the transition $\left|E_{i}\right\rangle \rightarrow\left|E_{f}\right\rangle$ occurs upon loss of a cavity photon [in the master equation (8), such transitions are triggered by the term $\left.\kappa \hat{a} \rho \hat{a}^{\dagger}\right]$. When the transition occurs, a photon is emitted into extra-cavity modes with (approximately) frequency $\omega_{f i}=E_{f}-E_{i}$ and bandwidth $\Gamma_{i}+\Gamma_{f}+\delta E_{i}+\delta E_{f}$. The $A_{i \rightarrow f}$ terms involving only JCM-type eigenstates do not present any particular surprises: They approximate the well-known JCM results for $\varepsilon \ll g_{\text {eff }}$, and converge to them for $\varepsilon \rightarrow 0$. We, thus, omit such cases for brevity and focus only on the matrix elements involving quasidark states, which yield the following expressions:

$$
\begin{aligned}
A_{1 D \rightarrow 0} & =\frac{\varepsilon^{2}}{4 g_{\text {eff }}^{2}+\varepsilon^{2}}, \\
A_{n D \rightarrow n-1, D} & =n-\frac{4 g_{\text {eff }}^{2} n}{4 g_{\text {eff }}^{2} n+\varepsilon^{2}}, \\
A_{n D \rightarrow n-1, \pm} & =0, \\
A_{n \pm \rightarrow n-1, D} & =\frac{2 g_{\text {eff }}^{2} \varepsilon^{2}}{\left[4 g_{\text {eff }}^{2}(n-1)+\varepsilon^{2}\right]\left(4 g_{\text {eff }}^{2} n+\varepsilon^{2}\right)} .
\end{aligned}
$$

From Eqs. (27)-(29) we note that, once the system is in one of the quasi-dark-states $\left|E_{n D}\right\rangle$, the loss of a cavity photon can only produce the quasi-dark-state $\left|E_{n-1, D}\right\rangle$ for $n \geqslant 2$ or $|G, 0\rangle$ for $n=1$. This corresponds to the emission of a photon at the cavity frequency $\omega_{0}$ with the associated matrix element of order $\sim n-1$. Assuming for the moment $\gamma_{d}=0$ (no dephasing), this implies that once the system has evolved into a quasidark state, it essentially reproduces the open dynamics of an empty cavity until state $|1, D\rangle$ is reached. From there the system will decay slowly towards the lowest state $|G, 0\rangle$, resulting again in the emission of a photon at the cavity frequency $\omega_{0}$ but with a much narrower bandwidth $\Gamma_{1 D} \simeq \kappa \varepsilon^{2} / 4 g_{\text {eff }}^{2}$. On the other hand, from Eq. (30) we can also see a small probability that a JCM-like state decays into a quasidark state by loss of a cavity photon. This process results in the emission of photons at average frequency $\omega_{0} \pm g_{\text {eff }} \sqrt{n}$ : These exactly overlap with the JCM spectral resonances for $n=1$, whereas they provide novel emission peaks for $n \geqslant 2$. Putting back finite dephasing $\gamma_{d} \neq 0$ into the picture, we see that, in addition to introducing further broadenings of the levels, dephasing events are capa- ble of interconverting states $|B\rangle$ and $|D\rangle$. More in detail, we find $|\langle f|L| i\rangle|^{2}=\gamma_{d} / 2$ for $L=\sqrt{\gamma_{d}}\left|e_{k}\right\rangle\left\langle e_{k}\right|$ with $k=1,2$ and $i, f \in\{D, B\}$. Referring again to the quantum jump approach [35] this will swap the states $|B\rangle$ and $|D\rangle$ with probability $1 / 2$ if a dephasing event occurs.

To summarize, we have provided a detailed characterization of the processes occurring in our open system for the emblematic case $N=2, \Delta=\Lambda=\gamma=\Omega=0$. Although the multilevel model can approximate all features of a JCM for $\varepsilon \ll g_{\text {eff }}$, it displays additional phenomena due to the indirect interaction of dark states and cavity field. In the next section we explore these issues quantitatively via numerical simulations.

\section{CLIMBING THE JC LADDER}

In the following numerical studies, we will investigate how closely the physics of our system-as relevant for typical quantum optics experiments-resembles a JCM with enhanced coupling constant $g_{\text {eff }} \propto \sqrt{N}$. For the sake of comparison, we will also display the behavior of a TC model with $N$ atoms and the same effective coupling constant $g_{\text {eff }}$-see the Appendix for further details. Simulations are performed for $N=3$ : This is one step up in complexity compared to the previous section but simple enough that the resulting calculations can be handled by a standard computer.

Our analysis focuses on two observables that are ubiquitous in both theoretical and experimental investigations of the JC model (proper definitions will be given below):

(i) The steady-state spectrum $S_{a}(\omega)$ of the cavity output field when the system is excited via incoherent pumping of the emitter.

(ii) The photon autocorrelation function $g^{(2)}(0)$ when the emitter is excited via a coherent driving field with variable detuning.

The first observable provides valuable information about the energy-level structure of a quantum system, in particular, highlighting its optically allowed transitions. Instead, $g^{(2)}(0)$ may be seen as a witness of optical nonlinearity: We will use it in our model to show that the $\sqrt{N}$ enhancement of the coupling constant $g_{\text {eff }} \propto \sqrt{N}$ does not come at the cost of "linearizing" the light-matter interaction (something that instead happens in the TC scenario as $N$ is increased, as we will see below). To support this last point, we will indeed compare our autocorrelation functions with $g^{(2)}(0)$ obtained for a reference coupled-oscillator model-i.e., the quintessential linear system in our context (see the Appendix for details). Note that we are focusing only on observables that can be measured via the cavity output field. The latter has indeed the same definition in all models considered here, facilitating direct comparisons between them. On the other hand, emitter observables do not have an unambiguous correspondence in the different models as they are defined on Hilbert spaces of different dimensions.

In detail, according to standard input-output theory [40], the portion of the cavity output field that reaches our detector, say $\hat{E}_{\text {out }}$ (scalar for simplicity), may be expressed as a function of the cavity annihilation operator $\hat{a}$ as follows:

$$
\hat{E}_{\mathrm{out}}=E_{\mathrm{c}} \hat{a}+\hat{E}_{\mathrm{vac}},
$$


where $E_{\mathrm{c}}$ is a constant depending on the details of the cavity-plus-detector setup, whereas the operator $\hat{E}_{\mathrm{vac}}$ captures all "vacuum noise" contributions from the detector's environment. Thanks to the assumption of a zero temperature environment for the cavity field, which is justified for optical frequencies at room temperature, $\hat{E}_{\mathrm{vac}}$ does not contribute to any normally ordered observable constructed from $\hat{E}_{\text {out }}$-and these are precisely the types of observables employed here.

In the interest of brevity, we restrict ourselves to an experimentally desirable situation in which $\Delta=0$ and the hierarchy $\varepsilon \ll \kappa \lesssim g_{\text {eff }}$ holds. In other words we consider a strongly coupled system where the average transition frequency of the emitter is at resonance with the cavity, whereas the range of sublevel detunings is within the bare cavity bandwidth. This scenario is indeed appropriate for the case of quantum dots embedded in plasmonic nanoresonators as anticipated in Sec. II. We will also take the driving strengths ( $\Omega$ for the coherent and $\Lambda$ for the incoherent case) as the smallest parameters in the problem. This ensures that the driving will not induce significant modifications to the intrinsic resonances of the coupled system nor to the conditions for achieving strong coupling [4-6,41-47]. In this setting, we focus on two representative parameter regimes:

(i) $g_{\text {eff }}=\kappa$ or the "single-excitation regime,"

(ii) $g_{\text {eff }}=20 \kappa$ or the "biexcitation regime."

Loosely speaking, case (i) gives access to the first rung of the energy ladder, thus, the resulting Physics should be similar to that of a pair of coupled oscillators in all models. Case (ii) instead allows us to explore the "second rung" of the ladder where the anharmonic nature of the investigated models becomes more prominent. In both situations we will analyze the role of emitter dephasing by considering three scenarios: no dephasing, low dephasing (i.e., one order of magnitude smaller than the cavity decay rate $\kappa$ ), and high dephasing (i.e., dephasing and cavity decay rates of the same order). This is particularly relevant for quantum-dot implementations of our models especially at room temperature, where emitter dephasing rates can be comparable to the cavity decay rate. Finally it is important note that we will adopt a logarithmic scale in our plots, since the examined quantities display features of very different magnitudes. This implies that the weaker features displayed in our plots (e.g., the second-rung spectral resonances) may be challenging to detect in experiments.

\section{A. Steady-state spectra}

As anticipated, our first set of numerical examples investigates the optical emission properties of our multilevel model under incoherent pumping of the emitter, as relevant in many quantum dot experiments also at ambient conditions. Specifically, we set $\Omega=0$ (no coherent driving) and $\Lambda \neq 0$. In this setting we are interested in the steady-state spectrum of the cavity output field, i.e.,

$$
\begin{aligned}
S_{a}(\omega) & =2 \operatorname{Re}\left(\int_{0}^{\infty}\left\langle\hat{E}_{\text {out }}^{\dagger}(\tau) \hat{E}_{\mathrm{out}}(0)\right\rangle_{\infty} e^{-i \omega \tau} d \tau\right), \\
& \propto 2 \operatorname{Re}\left(\int_{0}^{\infty}\left\langle\hat{a}^{\dagger}(\tau) \hat{a}(0)\right\rangle_{\infty} e^{-i \omega \tau} d \tau\right),
\end{aligned}
$$

where Eq. (31) has been used, the two-point correlation function $\left\langle\hat{a}^{\dagger}(\tau) \hat{a}(0)\right\rangle_{\infty}$ is calculated via the quantum regression theorem [35], and the expectation value is calculated on $\rho_{\infty}$, the steady state of the master equation. The latter is found by solving the linear system $\mathcal{L} \rho_{\infty}=0, \operatorname{Tr}\left[\rho_{\infty}\right]=1$ where the superoperator $\mathcal{L}$ is implicitly defined by rewriting the master equation (8) as $\dot{\rho}=\mathcal{L} \rho$. We perform such calculations numerically with PYTHON by truncating the Hilbert space of the cavity to a sufficiently high dimension to obtain numerical convergence. In addition to the cavity spectrum, we will also be interested in the quantity

$$
\begin{aligned}
p_{\text {dark }} & =\sum_{k} \operatorname{Tr}\left[\rho_{\infty}\left|D_{k}\right\rangle\left\langle D_{k}\right|\right] \\
& =1-\operatorname{Tr}\left[\rho_{\infty}(|B\rangle\langle B|+| G\rangle\langle G|)\right],
\end{aligned}
$$

i.e., the steady-state probability that the emitter has settled in a dark state (or a mixture thereof). The resulting spectra, on the logarithmic scale, are plotted in Fig. 5 together with those of the reference TC and JC models (again, see the Appendix for details). For a fairer visual comparison among the three models we have also rescaled the spectrum of JC and TC models by a factor of $1-p_{\text {dark }}$. We indeed recall from Sec. IV A that the multilevel model cannot display JC physics once it gets "stuck" into a dark state.

In the single-excitation regime [Figs. 5(a)-5(c)], we find that all three models display the expected resonances at $\omega \simeq$ $\omega_{0} \pm g_{\text {eff }}$, but the multilevel model presents additional sharp features at $\omega \simeq \omega_{0}$. In the case of no dephasing, these resonances account for a significant portion of the total emitted energy, and, correspondingly, $p_{\text {dark }}$ is close to one. We argue that the $\omega \simeq \omega_{0}$ features can be ascribed to the slow decay of the long-lived quasidark states of the single-excitation sector: Referring to our discussion and notation in Sec. IV A, this involves transitions of the form $\left|E_{1 D}\right\rangle \rightarrow|G, 0\rangle$ (where $\left|E_{1 D}\right\rangle$ can be any of the two quasidark states occurring for $N=3$ ). As dephasing is increased, going left to right in Fig. 5, we find that the three models show increasing agreement, and at the same time $p_{\text {dark }}$ is significantly reduced. Referring again to our analytical discussion in Sec. IV A, we recall that dephasing is indeed able to swap bright and dark states. This opens a new decay channel for the quasi-dark-states $\left|E_{1 D}\right\rangle$ : As dephasing is increased, we may reach a parameter regime where the quasi-dark-states $\left|E_{1 D}\right\rangle$ are more likely to decay by a process of the form $\left|E_{1 D}\right\rangle \rightarrow\left|E_{1 \pm}\right\rangle \rightarrow|G, 0\rangle$ where the first step is due to dephasing, and the second step is due to photon emission. Hence, in such conditions JC-like features can become more prominent in the measured spectrum. At high dephasing $\left[\gamma_{d}=\kappa\right.$, Fig. 5(c)], the dark-state features are effectively invisible in the emission spectrum.

The biexcitation scenario [Figs. 5(d)-5(f)] is where the multilevel model truly shines, compared to the reference TC model: We can see in the bottom half of Fig. 5 that multilevel and JC models are indeed in agreement on the location of both single-excitation ( $\omega \simeq \omega_{0} \pm g_{\text {eff }}$, gray dot-dashed lines) and biexcitation $\left[\omega \simeq \omega_{0} \pm(\sqrt{2} \pm 1) g_{\text {eff }}\right.$, gray dotted lines] resonances, whereas the biexcitation peaks of the TC model are visibly shifted. Also in this case the multilevel model presents an additional resonance (or perhaps a collection of closely spaced resonances) at $\omega \simeq \omega_{0}$. Referring again to the 

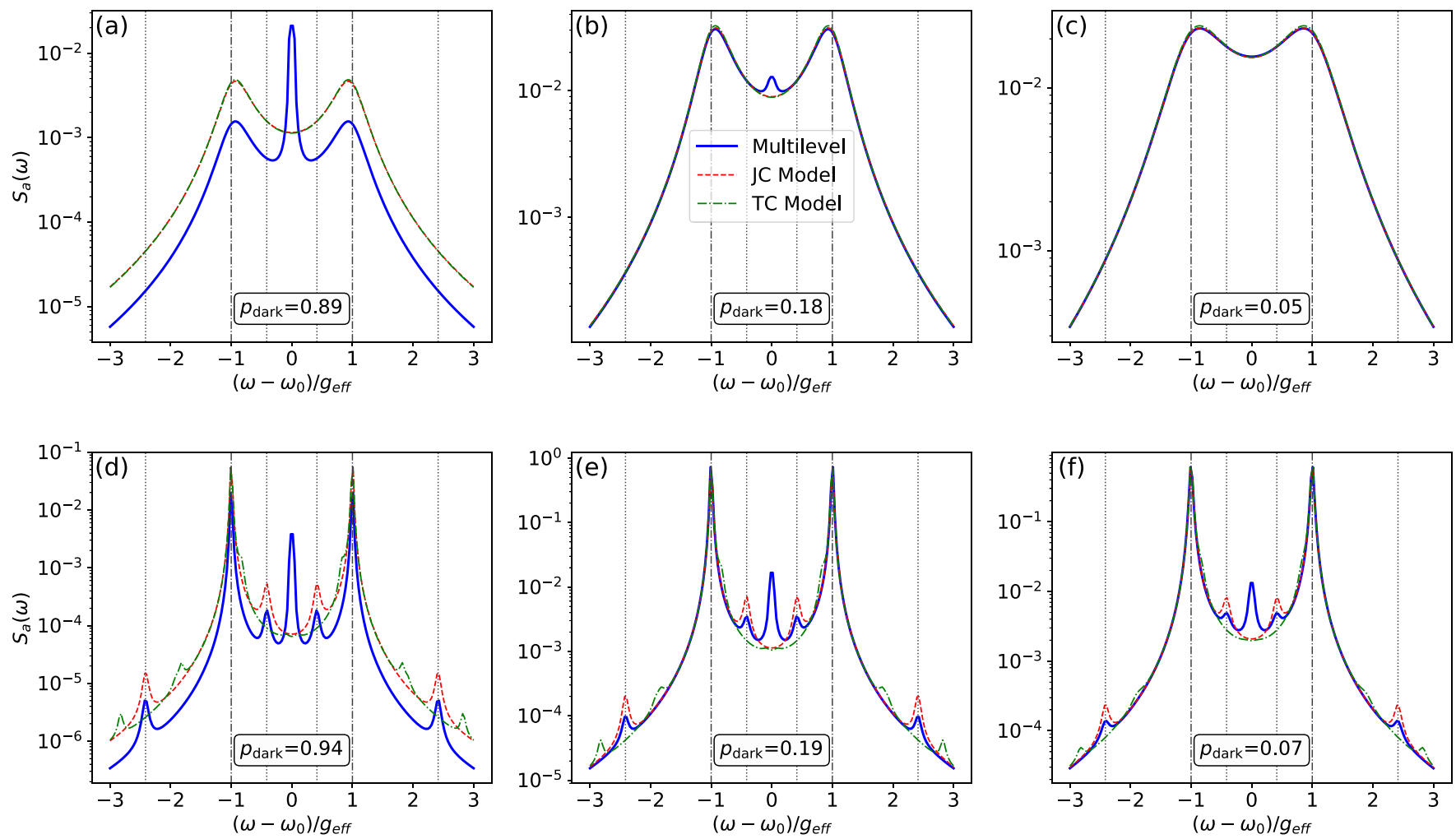

FIG. 5. Comparison of cavity emission spectra under incoherent pumping $(\Lambda \neq 0, \Omega=0)$. Spectra $S_{a}(\omega)$ are plotted for the multilevel model with $N=3, \varepsilon=0.05 g_{\text {eff }}$ (solid blue), as well as reference JC (dashed red) and TC models (dot-dashed green). Plots (a)-(c) are in the single-excitation regime with $\kappa=g_{\text {eff }}$ and $\Lambda=0.01 \kappa$. Plots (d)-(f) are in the biexcitation regime with $\kappa=0.05 g_{\text {eff }}$ and $\Lambda=0.02 \kappa$. Dephasing increases from left to right: (a) $\gamma_{d}=0$, (b) $\gamma_{d}=0.1 \kappa$, (c) $\gamma_{d}=\kappa$, (d) $\gamma_{d}=0$, (e) $\gamma_{d}=0.2 \kappa$, and (f) $\gamma_{d}=\kappa$. Spectra for the TC and JC models are rescaled by a factor $\left(1-p_{\text {dark }}\right)$. Gray vertical lines indicate the resonances corresponding to the first (dot dashed) and second rung (dotted) of the JC ladder. See the Appendix for further details on the TC and JC models employed here.

notation of Sec. IV A, these can be ascribed to two processes: The photons emitted in the $\left|E_{1 D}\right\rangle \rightarrow|G, 0\rangle$ transition, which is associated with a small decay rate, and those emitted in the second-rung process $\left|E_{2 D}\right\rangle \rightarrow\left|E_{1 D}\right\rangle$, which we recall is very similar to photon emission by an empty cavity and, hence, has a significantly higher decay rate $\simeq \kappa$. We again note that the $\omega \simeq \omega_{0}$ resonance can be weakened in the presence of dephasing, however, it remains prominent even in the high dephasing scenario [Fig. 5(f)]. Our interpretation for this is the following: Although dephasing of order $\kappa$ can suppress the $\left|E_{1 D}\right\rangle \rightarrow|G, 0\rangle$ process, as discussed above, the $\left|E_{2 D}\right\rangle \rightarrow$ $\left|E_{1 D}\right\rangle$ decay channel remains a probable process since it is also associated with a decay rate of order $\kappa$.

It is worth pointing out that if one goes beyond our symmetric pump hypothesis (populating all the emitter excited states with equal probability-see Sec. III), it may be possible to reduce the value of $p_{\text {dark }}$ and, hence, the prominence of dark-state features in the cavity emission spectrum.

To summarize, the results of this subsection tell us that the multilevel model is a viable system to test the characteristic $\propto \sqrt{n} g_{\text {eff }}$ splitting of energy levels that is still the holy grail of experimental JC research. In this light, the main strength of the multilevel model is that the effective coupling $g_{\text {eff }}$ may be significantly boosted by considering emitters with many closely spaced sublevels.

\section{B. Photon autocorrelation function}

As a second step, we will look at the steady-state behavior of the zero-delay photon autocorrelation function, under weak coherent driving of the cavity. In all the models employed here this quantity is defined as follows:

$$
\begin{aligned}
g^{(2)}(0) & =\lim _{t \rightarrow \infty} \frac{\left\langle\hat{E}_{\mathrm{out}}^{\dagger} \hat{E}_{\mathrm{out}}^{\dagger} \hat{E}_{\mathrm{out}} \hat{E}_{\mathrm{out}}\right\rangle}{\left\langle\hat{E}_{\mathrm{out}}^{\dagger} \hat{E}_{\mathrm{out}}\right\rangle^{2}} \\
& =\lim _{t \rightarrow \infty} \frac{\left\langle\hat{a}^{\dagger} \hat{a}^{\dagger} \hat{a} \hat{a}\right\rangle}{\left\langle\hat{a}^{\dagger} \hat{a}\right\rangle^{2}},
\end{aligned}
$$

where again we made use of Eq. (31) in order to obtain an expression that only features system operators. In detail, in this subsection we consider the master equation (8), setting $\Lambda=0, \Omega \neq 0$, and we will vary the driving field frequency $\omega_{L}$. Although the resulting master equation is explicitly time dependent, it is still possible to define a steady state by considering an interaction picture with respect to the Hamiltonian $H_{L}=\omega_{L} \hat{a}^{\dagger} \hat{a}+\omega_{L} \sum_{k=1}^{N}\left|e_{k}\right\rangle\left\langle e_{k}\right|$. This is a standard procedure in quantum optics, and it amounts to replacing $V_{\text {drive }} \rightarrow$ $V_{\text {drive }}^{\text {(int) }}=\Omega\left(\hat{a}+\hat{a}^{\dagger}\right)$ and $H \rightarrow H^{\text {(int) }}=H-H_{L}$ in the master equation (8). In turn, this procedure implicitly defines a timeindependent superoperator $\mathcal{L}^{(\text {int })}$ which does admit a steady state $\rho_{\infty}^{(\text {int })}$. Such a steady state is again found numerically with the same process described in the previous section, and it can 

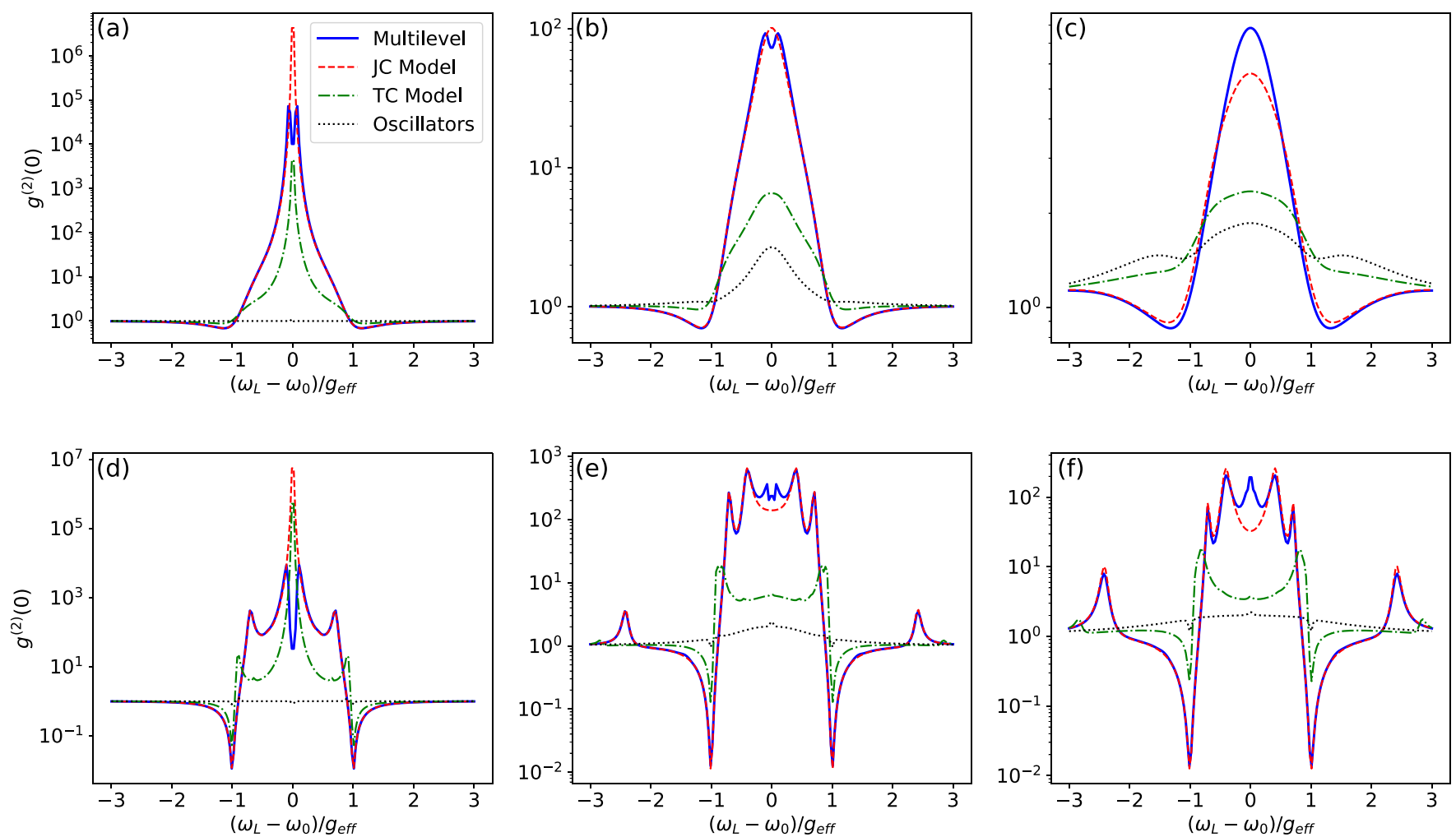

FIG. 6. Steady-state photon autocorrelation, $g^{(2)}(0)$, versus driving field detuning. Results are plotted for the multilevel model with $N=$ $3, \varepsilon=0.05 g_{\text {eff }}$ (solid blue), reference JC (dashed red), TC (dot-dashed green), and coupled-oscillator models (gray dotted). Driving strengths are set to $\Lambda=0, \Omega=0.01 g_{\text {eff }}$ in all cases. Plots (a)-(c) are in the single-excitation regime with $\kappa=g_{\text {eff }}$. Plots (d) $-(\mathrm{f})$ are in the biexcitation regime with $\kappa=0.05 g_{\text {eff }}$. Dephasing increases from left to right as in Fig. 5: Dephasing values are (a) $\gamma_{d}=0$, (b) $\gamma_{d}=0.1 \kappa$, (c) $\gamma_{d}=\kappa$, (d) $\gamma_{d}=0$, (e) $\gamma_{d}=0.2 \kappa$, and (f) $\gamma_{d}=\kappa$. See the Appendix for further details on JC, TC, and coupled-oscillator models.

be easily shown that it provides the correct expectation values involved in Eq. (34). The obtained autocorrelation functions for multilevel, JC, TC, and coupled-oscillator models are displayed in Fig. 6 as functions of the driving field detuning. We note that in this case we do not need to rescale JC, TC, and coupled-oscillator plots since $g^{2}(0)$ is normalized by definition.

We observe that, excluding the region $\omega \simeq \omega_{0}$, the multilevel model does a good job at matching the JC model predictions, capturing both the position and the magnitude of resonances and dips. These observations are true both in the single- and in the biexcitation regimes. In contrast, $g^{(2)}(0)$ of the TC model displays features that are significantly less pronounced than their JC counterparts (recall that we are using a logarithmic scale) as well as being shifted in frequency in the biexcitation regime. This is compatible with the intuition that the TC model is "more linear" than both the JC and the multilevel ones. Finally, the coupled-oscillator model is, of course, even farther off from the JC predictions: it indeed displays features that are several orders of magnitude smaller than those in all the other models. Additionally, the coupled-oscillator model satisfies $g^{(2)}(0)>1$ at all times. As anticipated, however, the multilevel model displays additional features in the region $\omega \simeq \omega_{0}$ : At low or no dephasing a double-peak structure can be clearly seen, likely due to processes involving the quasidark states (we recall that for $N=3$ there are two quasidark states for each value of the excitation number $n \geqslant 1$ ). As dephasing is increased, the prominence of these peaks is reduced, presumably due to the introduction of additional decay channels for the quasidark states (see also the previous subsection). However, dephasing also results in the appearance of a central peak in $g^{(2)}(0)$, which may be due to an increased likelihood that two driving photons excite a quasidark state of the system and are later emitted at the frequency of the empty cavity by a process of the form $\left|E_{2 D}\right\rangle \rightarrow\left|E_{1 D}\right\rangle \rightarrow|G, 0\rangle$. Although a more rigorous analysis of these effects would be certainly worthwhile to confirm our intuitions, we feel that it goes beyond the scope of this paper.

It is also interesting to note the stronger photon antibunching effect observed in the biexcitation regime (as compared to the single-excitation regime). This occurs around the frequencies of the two lowest excited states $E_{1, \pm}$. The biexcitation regime is indeed achieved by a higher quality system where the light-matter coupling is significantly larger than the decoherence and dephasing rates. In such conditions, the JC and multilevel models yield a strong nonlinear effect known as "photon blockade": The coupled light-matter system can only absorb or emit one photon at a time, causing the pronounced antibunching in the emitted field [40].

As anticipated, $g^{(2)}(0)$ is one of the most commonly adopted witnesses of nonlinearity in emitter-cavity systems, such as the ones we are investigating. The results of this subsection, therefore, confirm that the multilevel model closely matches the nonlinear signatures of JC physics, and in this respect it clearly outperforms the reference TC model with the same values of $N$ and $g_{\text {eff }}$. 


\section{CONCLUSIONS}

We explored a novel route to realize the strong coupling regime of light-matter interaction, exploiting a multilevel emitter coupled to a single-mode electromagnetic field. When the $N$ excited sublevels of the emitter are nearly degenerate, we showed both analytically and numerically that our proposed system can closely approximate many aspects of JC physics. The associated light-matter coupling constant scales as $g_{\text {eff }} \propto \sqrt{N}$ and, crucially, the quantum nonlinearities of our model are not suppressed with increasing $N$. Indeed, we are able to observe the characteristic energy-level splitting of or$\operatorname{der} \sim \sqrt{n}$, where $n$ is the number of combined (emitter + field) excitations, as well as the associated nonlinear signatures in emission spectra and photon autocorrelation functions. Moreover, we have been able to characterize the main effects associated with the additional levels of the emitter: The appearance of very interesting sharp resonances in the middle of the emission spectrum, associated with the slow decay of quasidark states. Although it may be possible to observe such resonances in an experiment, our analysis shows that these are quickly washed out in the presence of dephasing. Observing dark-state signatures would, thus, require cryogenic temperatures.

Our findings are particularly relevant in solid-state and plasmonic implementations of cavity QED, where the emitters of choice are often multilevel quantum dots, and may pave the way towards the observation and exploitation of light-matter strong coupling at room temperature.

\section{ACKNOWLEDGMENTS}

We thank M. G. Genoni for the useful discussion on modeling dephasing. We thank T. Fernholtz and J. H. Mueller for suggesting some important references. Support by the Science Foundation Ireland (SFI) under Grant No. 18/RP/6236 is gratefully acknowledged. T.T. acknowledges support from the University of Nottingham via a Nottingham Research Fellowship. This publication was supported by the Open Access Publication Fund of the University of Wuerzburg. D.F. acknowledges financial support from the DFG via grant HE5618/6-1.

\section{APPENDIX: AUXILIARY MODELS}

Here we briefly list the Hamiltonians and Lindblad operators for the auxiliary models used throughout the paper. In all cases the field mode is described by the same annihilation operator $\hat{a}$ (and corresponding Hilbert space) as in the main text. In turn, photon loss and coherent cavity driving are described by the same operators shown in the main text (Sec. III). What changes between the different models is the structure of the emitter and the associated Lindblad operators.

\section{Jaynes-Cummings model}

The Hamiltonian of the JC model adopted in the main text reads

$$
H_{\mathrm{JC}}=\omega_{0}\left(\hat{a}^{\dagger} \hat{a}+|e\rangle\langle e|\right)+g_{\mathrm{eff}}\left(\hat{a}^{\dagger}|g\rangle\langle e|+\hat{a}| e\rangle\langle g|\right),
$$

where the emitter is a two-level system with excited-state $|e\rangle$ and ground-state $|g\rangle$. The Lindblad operators for emitter decay, dephasing, and incoherent pumping are, respectively,

$$
\begin{aligned}
& L=\sqrt{\gamma}|g\rangle\langle e|, \\
& L=\sqrt{\gamma_{d}}|e\rangle\langle e|, \\
& L=\sqrt{\Lambda}|e\rangle\langle g|
\end{aligned}
$$

\section{Tavis-Cummings model with $N$ atoms}

The TC model used in the main text with $N$ atoms and effective coupling $g_{\text {eff }}$ reads

$$
H_{\mathrm{TC}}=\omega_{0} \hat{a}^{\dagger} \hat{a}+\omega_{0} \frac{\hat{J}_{z}}{2}+\frac{g_{\text {eff }}}{\sqrt{N}}\left(\hat{J}^{+} \hat{a}+\hat{a}^{\dagger} \hat{J}^{-}\right),
$$

where $\hat{J}_{x}, \hat{J}_{y}$, and $\hat{J}_{z}$ are spin- $N / 2$ operators and $\hat{J}^{ \pm}=\hat{J}_{x} \pm$ $i \hat{J}_{y}$ are the spin-ladder operators. The Lindblad operators for emitter decay, dephasing, and incoherent pumping, respectively, are

$$
\begin{aligned}
& L=\sqrt{\gamma} \hat{J}^{-}, \\
& L=\sqrt{\gamma_{d}} \hat{J}_{z} / 2, \\
& L=\sqrt{\Lambda} \hat{J}^{+} .
\end{aligned}
$$

\section{Coupled-oscillators model}

In the rotating-wave approximation, the Hamiltonian of two coupled harmonic oscillators (both having the same frequency) reads

$$
H_{\mathrm{osc}}=\omega_{0}\left(\hat{a}^{\dagger} \hat{a}+\hat{b}^{\dagger} \hat{b}\right)+g_{\mathrm{eff}}\left(\hat{a}^{\dagger} \hat{b}+\hat{b}^{\dagger} \hat{a}\right),
$$

where $\hat{a}, \hat{b}$ are annihilation operators of distinct Bosonic modes. The Lindblad operator for emitter decay, dephasing, and incoherent pumping, respectively, are

$$
\begin{aligned}
& L=\sqrt{\gamma} \hat{b}, \\
& L=\sqrt{\gamma_{d}} \hat{b}^{\dagger} \hat{b}, \\
& L=\sqrt{\Lambda} \hat{b}^{\dagger} .
\end{aligned}
$$

[1] B. W. Shore and P. L. Knight, The Jaynes-Cummings model, J. Mod. Opt. 40, 1195 (1993).

[2] S. Haroche and J.-M. Raimond, Exploring the Quantum: Atoms, Cavities, and Photons (Oxford Scholarship online, 2006).

[3] A. Reiserer, N. Kalb, G. Rempe, and S. Ritter, A quantum gate between a flying optical photon and a single trapped atom, Nature (London) 508, 237 (2014).
[4] M. Brune, S. Haroche, J. M. Raimond, L. Davidovich, and N. Zagury, Manipulation of photons in a cavity by dispersive atom-field coupling: Quantum-nondemolition measurements and generation of "Schrödinger cat" states, Phys. Rev. A 45, 5193 (1992).

[5] C. K. Law and J. H. Eberly, Arbitrary Control of a Quantum Electromagnetic Field, Phys. Rev. Lett. 76, 1055 (1996). 
[6] D. J. Wineland, Nobel lecture: Superposition, entanglement, and raising Schrödinger's cat, Rev. Mod. Phys. 85, 1103 (2013).

[7] P. Biagioni, J.-S. Huang, and B. Hecht, Nanoantennas for visible and infrared radiation, Rep. Prog. Phys. 75, 024402 (2012).

[8] H. Walther, B. T. Varcoe, B.-G. Englert, and T. Becker, Cavity quantum electrodynamics, Rep. Prog. Phys. 69, 1325 (2006).

[9] P. Törmä and W. L. Barnes, Strong coupling between surface plasmon polaritons and emitters: a review, Rep. Prog. Phys. 78, 013901 (2015).

[10] D. S. Dovzhenko, S. V. Ryabchuk, Y. P. Rakovich, and I. R. Nabiev, Light-matter interaction in the strong coupling regime: configurations, conditions, and applications, Nanoscale 10, 3589 (2018).

[11] X. Yu, Y. Yuan, J. Xu, K.-T. Yong, J. Qu, and J. Song, Strong coupling in microcavity structures: Principle, design, and practical application, Laser Photonics Rev. 13, 1800219 (2019).

[12] G. Zengin, G. Johansson, P. Johansson, T. J. Antosiewicz, M. Käll, and T. Shegai, Approaching the strong coupling limit in single plasmonic nanorods interacting with J-aggregates, Sci. Rep. 3, 3074 (2013).

[13] M. Tavis and F. W. Cummings, Exact solution for an nmolecule-radiation-field hamiltonian, Phys. Rev. 170, 379 (1968).

[14] N. Quesada, Strong coupling of two quantum emitters to a single light mode: The dissipative Tavis-Cummings ladder, Phys. Rev. A 86, 013836 (2012).

[15] R. H. Dicke, Coherence in spontaneous radiation processes, Phys. Rev. 93, 99 (1954).

[16] M. Wersäll, J. Cuadra, T. J. Antosiewicz, S. Balci, and T. Shegai, Observation of mode splitting in photoluminescence of individual plasmonic nanoparticles strongly coupled to molecular excitons, Nano Lett. 17, 551 (2017).

[17] D. G. Baranov, B. Munkhbat, E. Zhukova, A. Bisht, A. Canales, B. Rousseaux, G. Johansson, T. J. Antosiewicz, and T. Shegai, Ultrastrong coupling between nanoparticle plasmons and cavity photons at ambient conditions, Nat. Commun. 11, 2715 (2020).

[18] M. Geisler, X. Cui, J. Wang, T. Rindzevicius, L. Gammelgaard, B. S. Jessen, P. A. D. Gonçalves, F. Todisco, P. Bøggild, A. Boisen, M. Wubs, N. A. Mortensen, S. Xiao, and N. Stenger, Single-crystalline gold nanodisks on WS2 mono- and multilayers for strong coupling at room temperature, ACS Photonics 6 , 994 (2019).

[19] A. J. Moilanen, T. K. Hakala, and P. Tćrmä, Active control of surface plasmon-emitter strong coupling, ACS Photonics 5, 54 (2018).

[20] K. Santhosh, O. Bitton, L. Chuntonov, and G. Haran, Vacuum Rabi splitting in a plasmonic cavity at the single quantum emitter limit, Nat. Commun. 7, ncomms11823 (2016).

[21] R. Chikkaraddy, B. de Nijs, F. Benz, S. J. Barrow, O. A. Scherman, E. Rosta, A. Demetriadou, P. Fox, O. Hess, and J. J. Baumberg, Single-molecule strong coupling at room temperature in plasmonic nanocavities, Nature (London) 535, 127 (2016).

[22] H. Groß, J. M. Hamm, T. Tufarelli, O. Hess, and B. Hecht, Near-field strong coupling of single quantum dots, Sci. Adv. 4, eaar4906 (2018).

[23] H. Leng, B. Szychowski, M.-C. Daniel, and M. Pelton, Strong coupling and induced transparency at room temperature with single quantum dots and gap plasmons, Nat. Commun. 9, 4012 (2018).

[24] K.-D. Park, M. A. May, H. Leng, J. Wang, J. A. Kropp, T. Gougousi, M. Pelton, and M. B. Raschke, Tip-enhanced strong coupling spectroscopy, imaging, and control of a single quantum emitter, Sci. Adv. 5, eaav5931 (2019).

[25] J. Kasprzak, S. Reitzenstein, E. A. Muljarov, C. Kistner, C. Schneider, M. Strauss, S. Höfling, A. Forchel, and W. Langbein, Up on the Jaynes-Cummings ladder of a quantumdot/microcavity system, Nature Mater. 9, 304 (2010).

[26] J. M. Fink, M. Göppl, M. Baur, R. Bianchetti, P. J. Leek, A Blais, and A. Wallraff, Climbing the Jaynes-Cummings ladder and observing its nonlinearity in a cavity QED system, Nature (London) 454, 315 (2008).

[27] J. P. Reithmaier, G. Sęk, A. Löffler, C. Hofmann, S. Kuhn, S. Reitzenstein, L. V. Keldysh, V. D. Kulakovskii, T. L. Reinecke, and A. Forchel, Strong coupling in a single quantum dotsemiconductor microcavity system, Nature (London) 432, 197 (2004).

[28] K. H. Madsen, T. B. Lehmann, and P. Lodahl, Role of multilevel states on quantum-dot emission in photonic-crystal cavities, Phys. Rev. B 94, 235301 (2016).

[29] H. Liu and P. Guyot-Sionnest, Photoluminescence lifetime of lead selenide colloidal quantum dots, J. Phys. Chem. C 114, 14860 (2010).

[30] Z. Hens and I. Moreels, Light absorption by colloidal semiconductor quantum dots, J. Mater. Chem. 22, 10406 (2012).

[31] C. Tserkezis, A. I. Fernández-Domínguez, P. A. D. Gonçalves, F. Todisco, J. D. Cox, K. Busch, N. Stenger, S. I. Bozhevolnyi, N. A. Mortensen, and C. Wolff, On the applicability of quantum-optical concepts in strong-coupling nanophotonics, Rep. Prog. Phys. 83, 082401 (2020).

[32] N. Kongsuwan, A. Demetriadou, M. Horton, R. Chikkaraddy, J. J. Baumberg, and O. Hess, Plasmonic nanocavity modes: From near-field to far-field radiation, ACS Photonics 7, 463 (2020).

[33] S. Franke, S. Hughes, M. K. Dezfouli, P. T. Kristensen, K. Busch, A. Knorr, and M. Richter, Quantization of Quasinormal Modes for Open Cavities and Plasmonic Cavity Quantum Electrodynamics, Phys. Rev. Lett. 122, 213901 (2019).

[34] A. F. Kockum, A. Miranowicz, S. De Liberato, S. Savasta, and F. Nori, Ultrastrong coupling between light and matter, Nat. Rev. Phys. 1, 19 (2019).

[35] H. P. Breuer and F. Petruccione, The Theory of Open Quantum Systems (Oxford University Press, Oxford, 2002).

[36] A nonzero (but small) $\gamma$ provides a negligible contribution to the cavity emission properties of the system. If one were to observe the direct emitter emission, however, this would be dependent on $\gamma$. In particular, for $\gamma=0$ there would be no direct emitter emission into "laboratory" field modes. On the other hand, for nonzero $\gamma$ a very small portion of the detectable radiation (on the order of $\gamma / \kappa$ ) would be coming directly from the emitter. The specific order of magnitude of $\gamma$ depends on the particular implementation. In quantum dots, it is typical to have $\gamma$ several orders of magnitude smaller than both the cavity decay rate and the emitter-cavity coupling constant so that the effect of direct emitter radiation into external modes is negligible for all practical purposes (particularly, when cavity output fields are detected). For example, the CdSe quantum dots used in Ref. [22] had a radiative decay rate on the order 
of $10^{7} \mathrm{~Hz}$ ), whereas the low- $q$ cavity employed there had a far greater decay rate on the order of $10^{13} \mathrm{~Hz}$.

[37] M. O. Scully, Correlated Spontaneous-Emission Lasers: Quenching of Quantum Fluctuations in the Relative Phase Angle, Phys. Rev. Lett. 55, 2802 (1985).

[38] D. T. Pegg, R. Loudon, and P. L. Knight, Correlations in light emitted by three-level atoms, Phys. Rev. A 33, 4085 (1986).

[39] P. Knight, S. Barnett, B. Dalton, M. Kim, F. de Oliveira, and K. Wodkiewicz, Dynamics of three-level atoms: jumping and squeezing, Fundamentals of Quantum Optics II (Springer, Heidelberg, 2005), pp. 71-91.

[40] D. F. Walls and G. J. Milburn, Quantum Optics (Springer, Heidelberg, 2008).

[41] F. P. Laussy, E. del Valle, and C. Tejedor, Luminescence spectra of quantum dots in microcavities. I. Bosons, Phys. Rev. B 79, 235325 (2009).

[42] E. del Valle, F. P. Laussy, and C. Tejedor, Luminescence spectra of quantum dots in microcavities. II. Fermions, Phys. Rev. B 79, 235326 (2009).
[43] G. Zengin, M. Wersäll, S. Nilsson, T. J. Antosiewicz, M. Käll, and T. Shegai, Realizing Strong Light-Matter Interactions between Single-Nanoparticle Plasmons and Molecular Excitons at Ambient Conditions, Phys. Rev. Lett. 114, 157401 (2015).

[44] Y. Sugawara, T. A. Kelf, J. J. Baumberg, M. E. Abdelsalam, and P. N. Bartlett, Strong Coupling between Localized Plasmons and Organic Excitons in Metal Nanovoids, Phys. Rev. Lett. 97, 266808 (2006).

[45] A. E. Schlather, N. Large, A. S. Urban, P. Nordlander, and N. J. Halas, Near-field mediated plexcitonic coupling and giant rabi splitting in individual metallic dimers, Nano Lett. 13, 3281 (2013).

[46] D. G. Baranov, M. Wersäll, J. Cuadra, T. J. Antosiewicz, and T. Shegai, Novel nanostructures and materials for strong lightmatter interactions, ACS Photonics 5, 24 (2017).

[47] Y. Huang, F. Wu, and L. Yu, Rabi oscillation study of strong coupling in a plasmonic nanocavity, New J. Phys. 22, 063053 (2020). 